The use of the Gaps-In-Noise test as an index of the enhanced left temporal cortical thinning associated with the transition between mild cognitive impairment and Alzheimer's disease. Vasiliki (Vivian) Iliadou 1 , Doris-Eva Bamiou 2 , Christos Sidiras 1 , Nikolaos P Moschopoulos , $_{1}$ Magda Tsolaki $_{3}$, Ioannis Nimatoudis ${ }_{1}$, Gail D. Chermak4

Affiliations

1. Clinical Psychoacoustics Lab, 3rd Psychiatric Department, Neuroscience Sector, Medical School, Aristotle University of Thessaloniki

2. UCL Ear Institute, London

3. 3rd Department of Neurology, Aristotle University of Thessaloniki

4. Department of Speech and Hearing Sciences, Washington State University

Health Sciences, Spokane, Washington

Corresponding author:

Vasiliki (Vivian) Iliadou

Assistant Professor of Psychoacoustics, Medical School, Aristotle University of Thessaloniki

Filellinon 6 N 751

PC 55236

Thessaloniki, Greece

+2310994739

email: viliad@auth.gr 


\section{Structured abstract}

Purpose: To evaluate auditory perception in a group of older adults diagnosed with mild cognitive impairment (MCI).

Method: A cross-sectional study of auditory perception in adults with MCI and adults with no documented cognitive issues and matched hearing sensitivity and age. Auditory perception was evaluated in both groups assessing for hearing sensitivity, speech in babble and temporal resolution.

Results: Mann-Whitney test revealed significantly poorer scores for Speech-in-Babble (SinB) and temporal resolution abilities of MCIs vs normal controls for both ears. The right ear gap detection thresholds on the Gaps-In-Noise (GIN) test clearly differentiated between the two groups $(\mathrm{p}<0.001)$, with no overlap of values. The left ear results also differentiated the two groups $(\mathrm{p}<0.01)$; however, there was a small degree of overlap around $8 \mathrm{msec}$ threshold values. With the exception of the left ear inattentiveness index which showed a similar distribution between groups, both impulsivity and inattentiveness indexes were higher for the MCIs compared to the control group.

Conclusions: The results support central auditory processing evaluation in the elderly population as a promising tool to achieve earlier diagnosis of dementia, while identifying central auditory processing deficits that can contribute to communication deficits in the MCI patient population. A measure of temporal resolution (GIN) may offer an early, albeit indirect measure reflecting left temporal cortical thinning associated with the transition between MCI and Alzheimer's disease. 


\section{Introduction}

Mild cognitive impairment (MCI) or mild neurocognitive disorder, as presented in the Diagnostic and Statistical Manual of Mental Disorders 5th edition (DSM-5), is diagnosed based on four criteria. These criteria include: 1) evidence of modest cognitive decline either as a concern or as a measured modest impairment in cognitive performance; 2) absence of interference with independence capacity for everyday activities; 3) cognitive deficits do not occur exclusively in the context of a delirium; and 4) deficits are not better explained by another mental disorder. MCI may lead to Alzheimer's disease (AD) in 10\%-15\% of cases. Several studies have documented auditory processing 
deficits in MCI, with specific deficits in speech in noise performance among the most often encountered deficit (Idrizbegovic et al, 2011; Rahman et al, 2011; Quaranta et al, 2015). A higher incidence (46.7\% as opposed to $4.6 \%$ ) of central auditory processing disorder (CAPD) was seen in the Framingham longitudinal population cohort in individuals who subsequently developed AD a decade later in life. This finding suggests the potential role of central auditory testing in the early diagnosis of AD (Gates et al, 1990).

A known link between auditory perception and cognition has been shown in studies of peripheral hearing loss (Lindenberger et al, 2001); however, it is often overlooked when testing for cognition (Dupuis et al, 2015). This is an important finding because adults with hearing loss may be misdiagnosed with MCI if their hearing loss is not tested prior to the MCI assessment (Jorgensen et al, 2016). Moreover, this misdiagnosis could lead to the withholding of hearing aids when fitting with hearing aids could actually change their performance on cognitive tests and improve their communication (Weinstein et al, 1986). Elderly population studies show an independent association between peripheral hearing impairment and reduced neurocognitive performance (Gussekloo et al, 2005; Lin, 2011). Hearing impaired adults have an increased risk of dementia (Gallacher et al, 2012) and accelerated rate of cognitive decline (Lin et al, 2013), as well as an accelerated decline in both temporal lobe regional volumes and whole brain volume (Lin et al, 2014). The observed association between auditory and cognitive deficits may be explained, at least in part, as a result of shared neuropathology, although social isolation resulting from hearing loss may also contribute to cognitive decline (Barnes et al, 2004; Bennett et al, 2006).

Speech recognition in competition or noise is typically deficient in Alzheimer's disease (Iliadou et al, 2003). Auditory perception is underpinned by hearing sensitivity, as measured by pure tone detection on audiometry. However, recognition and understanding of speech in backgrounds of noise is highly dependent on additional auditory processing skills that include temporal resolution, which is important for phoneme differentiation (e.g., discriminating between voice and voiceless stop 
consonants) and prosody skills (Chermak et al, 2005). In addition, perception of speech in competition and/or noise is dependent on working memory capacity (Ronnberg, 2003; Gatehouse et al, 2006; Akeroyd, 2008). Not surprisingly, peripheral hearing loss and central auditory processing deficits place increased demands on cognitive processing capacity (McCoy et al, 2005) due to the necessary reallocation of brain resources needed to support auditory perception and 'higher' level central auditory processes. The relationship seen between auditory and cognitive deficits may be explained, at least in part, as a result of shared neuropathology. In addition, social isolation resulting from hearing loss may also contribute to cognitive decline (Barnes et al, 2004; Bennett et al, 2006).

Temporal processes are crucial to a wide range of auditory and auditory-language behaviors including rhythm perception, periodicity pitch discrimination, duration discrimination, phoneme discrimination, and segregation of auditory figure and ground (i.e. listening in noise or competition) (Chermak \& Lee, 2005; Phillips, 2002). Chermak and Musiek (1997) emphasized the role of temporal processing across the range of language processing skills, from phonemic, voice-onset time differentiation underlying cognate discrimination (e.g., /b/ v. /p/), to lexical distinctions (e.g., "sub' ject" v. "sub ject'"), temporally cued prosodic distinctions (e.g., "night rate" v. "nitrate"), and resolution of ambiguity (e.g., "she saw the snow drift by the window" v. "she saw the snowdrift by the window"). Temporal resolution, referring to the ability to perceive time-related changes in ongoing auditory stimuli, underlies the discrimination of voiced from unvoiced stop consonants (e.g., Elangovan \& Stuart, 2008), and is commonly measured clinically using gap detection tests. The listener is required to detect a brief period of silence in an acoustic stimulus. Older adults typically are found to have worse gap detection performance with poorer (longer) gap thresholds than younger adults (Murphy, Dillon, Schochat, Musiek, Bamiou, 2016 personal communication). Moreover, older adults' gap duration difference limens increase more rapidly with frequency disparity between the center frequency of the leading marker and that of the trailing marker in a between-channel gap detection paradigm, a paradigm that represents the temporal cues in speech that involve sounds surrounded by other sounds of different spectral characteristics (Lister, Besing, and Koehnke, 2002). Impaired temporal acuity is 
reported in the elderly with normal hearing or hearing loss (Moore et al, 1992; Snell et al, 2000; Pichora-Fuller et al, 2003). As testing for temporal resolution usually involves rapid motor (Gaps-InNoise [GIN]) or verbal (Random Gap Detection Test) responses (Keith, 2000; Musiek et al, 2005), it is unclear whether the findings reflect general aging with consequent slowing of responses and/or true auditory processing deficits.

All the above mentioned studies show that the association between hearing and cognition is a complex one with both processes influencing one another in a way that is not always predictive. Hearing in everyday life extends way beyond hearing sensitivity, as measured by the pure tone audiogram, and auditory processing should be evaluated to complete the picture. Both attention and executive function (i.e. self-regulation required to respond appropriately to instructions) may interfere with auditory processing measurement. To minimize these aspects of cognition (attention and executive function) in the present study, within measurement variables were taken into account.

The goal of the present study was to examine peripheral (pure tone thresholds) and central aspects of auditory perception (temporal resolution and speech recognition in noise) in a group of older adults diagnosed with MCI and an age-matched control group. A secondary aim was to examine the role of attention by examining participants' responses to gaps that exceeded their gap thresholds, as well as false positive responses (i.e., when gaps are absent) potentially related to behavioral regulation issues.

\section{Material \& Methods}

Eighteen adults (12 females; 6 males) ranging in age between 51 and 82 years diagnosed with MCI participated in the present study. They were recruited from the memory and dementia clinic run by author M.T. Eleven participants ( 6 females and 5 males) ranging in age between 50 and 73 years, with no documented cognitive issues or complaints regarding cognition were assigned to the normal control group. MCI and control groups were matched for age, sex and average pure tone hearing 
thresholds. Specific results may be found in table 1 . The average pure tone hearing sensitivity of tested frequencies $250 \mathrm{~Hz}, 500 \mathrm{~Hz}, 1000 \mathrm{~Hz}, 2000 \mathrm{~Hz}, 4000 \mathrm{~Hz}, 8000 \mathrm{~Hz}$ was calculated for each ear (right and left).

MCI participants were examined with an assessment battery, consisting of a semi-structured interview (Clinical Dementia Rating - CDR) with a specialist in neurocognitive disorders, a scale for depression (Geriatric Depression Scale - GDS), a general medical and neurological examination and a neuropsychological assessment (Mini Mental State Examination - MMSE, Montreal Cognitive Assessment - MoCA). The diagnosis of MCI was assigned consistent with the DSM-5 criteria for Mild Neurocognitive Disorder (see introduction) and the recommendations of the International Working Group on Mild Cognitive Impairment (Winblad et al, 2004) specifying MCI criteria as absence of dementia diagnosis, evidence of cognitive deterioration substantiated by objective and/or subjective self and/or informant report and preservation of daily living activities. Current guidelines focus on clinical diagnosis and do not provide specific tests and cut-off criteria for MCI diagnosis. However, mean data from recent published papers are used in the clinical setting to further validate diagnosis.

The Clinical Dementia Rating (CDR) (Morris, 1993) uses a semi-structured interview to quantify the severity of symptoms of dementia. The subject's cognitive status is rated in 6 domains of functioning which include memory, orientation, judgment and problem solving, community affairs, home and hobbies, and personal care. The CDR-SB ( $\mathrm{SB}=\mathrm{Sum}$ of Boxes) score is obtained by summing each of the domain box scores, with scores ranging from 0 to 18 . Information is provided by both the patient and an informant. MCI scores typically fall (O'Bryant et al, 2010) at a mean of 1.3 $(\mathrm{sd}= \pm 1.16)$, with normal controls scoring at a mean of $0.11(\mathrm{sd}= \pm 0.36)$.

The Geriatric Depression Scale (GDS) (Yesavage et al, 1982) is a 30 items depression scale requiring "yes" or "no" patient responses to a series of questions (e.g., "Are you basically satisfied with your life?; Have you dropped many of your activities and interests?; Do you feel that your life is empty?"). One point is assigned to each answer and the cumulative score is rated on a scoring grid. Of the 15 items, 10 indicate the presence of depression when answered positively, while the rest 
(question numbers $1,5,7,11,13$ ) indicate depression when answered negatively. The grid sets a range of 0-9 as "normal", 10-19 as "mildly depressed", and 20-30 as "severely depressed". MCI participants were excluded from the study if they scored 10 or higher on the GDS.

The Mini Mental State Examination (MMSE) (Folstein et al, 1975) is a screening test used to assess general cognitive functioning. Areas of functioning assessed include orientation, registration, attention, recall, working memory, language, and construction or drawing ability. Possible scores range from 0 to 30. Patients diagnosed with MCI typically (O'Bryant et al, 2010) score at a mean of $27.2( \pm 2.27)$, with normal controls scoring at a mean of $28.9( \pm 1.29)$. As data of MCI and normal controls are so close this screening test is not to be used as a stand alone evaluation for differentiating MCI from normal controls.

The Montreal Cognitive Assessment (MoCA) (Nasreddine et al, 2005) is a newly developed cognitive screening instrument, with high sensitivity and specificity for MCI (Popovic et al, 2007; Hoops et al, 2009; Pendlebury et al, 2010). The MoCA assesses the following cognitive domains: visual spatial abilities, executive functions, short-term memory, attention, concentration, working memory, language and orientation. Possible scores range from 0 to 30. MCI patients typically score at a mean of $22.1(\mathrm{sd}= \pm 3.1)$ in contrast to normal controls whose typical score is at $27.4(\mathrm{sd}=2.2)$.

All 18 participants were tested on pure tone audiometry, speech recognition in quiet, and central auditory processing tests, including the speech in babble (SinB) (Iliadou et al, 2006; Sidiras et al, 2016) and auditory temporal resolution tests (GIN and RGDT). All tests were administered in a sound treated booth. The speech and temporal resolution tests were available on CD, played by a CD player through a GSI 61 audiometer and presented over TDH headphones at $60 \mathrm{~dB}$ HL. This presentation level was chosen in order to assess auditory perception as it relates to everyday average speech levels (Ferrand, 2006), as well as for comparison purposes across the two groups. Prior to testing, participants confirmed that the test sounds were comfortably audible.

The speech in quiet test was conducted monaurally using lists of 25 bisyllabic words from one of the three available phonetically balanced and frequently occurring words in spoken Greek 
(Iliadou et al, 2006). Each syllable correctly repeated was scored 2 percentage points.. The central auditory processing test battery of the study was chosen on the basis of guidelines of several professional associations (ASHA, 2005; AAA, 2010; BSA, 2011). It consisted of one verbal (the monaural low-redundancy Greek Speech in Babble test-SinB) and two non-verbal tests (RGDT and GIN). Of the two temporal resolution tests, the GIN is less cognitively demanding given its motor response compared to the RGDT that requires a verbal report that involves counting (one or two tones). Central auditory tests were administered in random order.

The Speech in Babble test (SinB) was developed in the Psychoacoustic Laboratory of the Aristotle University of Thessaloniki. It consists of two lists (one for each ear) of 50 phonetically balanced disyllabic words and background multitalker babble (Iliadou et al, 2006; Iliadou et al, 2009; Sidiras et al, 2016). The test was administered monaurally. Signal to noise ratio (SNR) varied within lists. Five SNRs $[+7,+5,+3,+1$ and -1$]$ were used in a fixed descending order [i.e. starting with the easiest listening condition (SNR +7) and finishing with the most difficult listening condition (SNR 1)] with each SNR applied to ten words in each list. The participant's task was to repeat the word after each presentation. Performance was measured using the basic Spearman-Karber formula: SNR of $50 \%$ correct speech identification $=\mathrm{i}+1 / 2(\mathrm{~d})-[(\mathrm{d}) \mathrm{x}(\#$ corrects $)] / \mathrm{w}$ (where $\mathrm{i}=$ initial presentation level in $\mathrm{dB}, \mathrm{d}=$ step size, $\mathrm{w}=$ number of items per decrement [per step]) (Sidiras et al, 2016; Iliadou, 2013). Each participant was tested with a 50 word list in each ear.

The RGDT (Keith, 2000) followed by the GIN (Musiek et al, 2005; Shinn et al, 2009) were administered to all participants at 60dB HL. The RGDT was presented binaurally following successful completion of a practice section. The practice as well as the main section consists of pairs of pure tones separated by silent intervals: silent intervals for the practice section start at $0 \mathrm{msec}$ and gradually increase to $40 \mathrm{msec}$. In the main section of the test, the silent intervals are presented in random order for each of the following pure tones: $500 \mathrm{~Hz}, 1000 \mathrm{~Hz}, 2000 \mathrm{~Hz}$, and $4000 \mathrm{~Hz}$, which are tested in sequence. A 4.5 second inter-trial interval is used to allow subjects time to respond. Nine trials are presented in the practice section and nine for each of the frequencies tested. Each trial for each pure- 
tone frequency is presented once with a unique silent interval (i.e. gap). A total of 36 trials are used to calculate the overall gap detection threshold. The participant's task is to report whether one or two sounds was heard. The threshold of gap detection is calculated for each frequency tested as the shortest time interval at which the participant reports perception of two tones. Averaging the gap detection threshold of each of the four frequencies tested provides the average gap detection threshold across frequencies.

The GIN was presented monaurally, starting with the practice section for each ear. The practice test consists of 10 trials with random presentation of varying durations of gaps in white noise. The GIN is comprised of four lists with 32 to 36 trials each: list 1 has 35 trials, list 2 has 32 , list 3 has 29 , and list 4 contains 36 trials. Each trial consists of 6 seconds of white noise with a 5 second intertrial interval. Duration and location of the gaps is varied. The duration of the gaps is $2,3,4,5,6,8$, 10, 12, 15, and 20 msec. Each gap occurs six times within each list. Each subject was told that they were going to hear noise in which there might be short gaps with no noise, that some gaps would be shorter than others, and that in some cases no gaps would be present. The participant was told to indicate when they detected a gap by pressing a button. After each ear had been tested, the number and percent of correct responses for each gap was calculated. Gap detection threshold was calculated as the shortest gap duration detected on at least four out of six gaps. False positives were noted and subtracted from the correct responses as follows: total score $=$ (total number of correct responsesfalse positives $) /$ the number of trials in the list $(29-36)$.

As noted above, the GIN places low cognitive demands to successfully complete the task and therefore provides a more specific measure of temporal processing. As such, this measure was used to examine and quantify within test attention and executive skills that might interact with other measures. Three variables were derived from the GIN test results: two representing an impulsivity index and one an inattentiveness index. The impulsivity indexes were computed as follows: Impulsivity1_Hits=False Hits or false alarms/Correct Hits or true positives and Impulsivity2_Total_Items=False Hits or false alarms/Total items. False alarms refer to those button pressing responses 
in instances where no gap is present. The inattentiveness index was computed as the number of gaps the participant failed to identify despite the fact that the duration of those gaps exceeded the participant's measured gap threshold divided by the total number of items presented above that participant's measured threshold.

\section{Statistical analysis}

All statistical analysis was performed using the statistical package SPSS 22. Data from most tests used in the study were non-normally distributed, since not all skewness and kurtosis $\mathrm{z}$ values ranged between -1.96 and +1.96 (Doane and Seward, 2002; Cramer and Howitt, 2004); therefore, non-parametric statistics were used. Both raw and derived variables were analyzed using the MannWhitney test and all correlations were assessed with the Spearman's rho correlation coefficient. Group results are provided in table 1.

\section{Results}

Statistical analysis verified no significant differences in age, pure tone audiometric thresholds, and speech in quiet, for both right and left ears, between the MCI and normal control groups (MannWhitney test for age $U=68.5, p>.05$; Mann-Whitney test for pure tone audiometric thresholds for the right ear $U=67, p>.05$ and for the left ear $U=85.5$, p>.5; Mann-Whitney test for speech in quiet for the right ear $U=113, p>.5$ and for the left ear $U=90.5, p>.5)$. As our data present in two distinct groups, we performed the nonparametric Mann-Whitney U test to check for statistically significant differences between groups (MCI group and normal control group) for each of the three central auditory processing tests (RGDT, SinB, and GIN).

The Mann-Whitney test revealed that the MCI group's SinB scores were significantly poorer than those of normal controls (figure 1) for both ears (median=.9 and .6 for right and left ears, respectively for MCI; median=.0 for both ears for normal controls; $\mathrm{U}=48.0$ and $\mathrm{U}=50.5$, both $\mathrm{p}<.05$ for right and left ears, respectively). The MCI group's temporal resolution as measured by RGDT was poorer 
(figure 2) than the normal controls (median=35.8 vs median=12.5 for MCI and normal group, respectively; $\mathrm{U}=30.0, \mathrm{p}<.005$ ). The same finding was seen for GIN threshold scores (figure 3 ) for both ears (median=10 and 11 for right and left ears, respectively for MCI; median=8 for both ears for normal controls; $U=12.5$ and $U=23.0$, both $\mathrm{p}<.001$ and $\mathrm{p}<.01$ for right and left ears, respectively). Pure tone audiometry, speech in quiet, age and auditory processing evaluation results are shown in table 1 for the MCI and control groups, as well as for a subgroup of the MCI and control groups exhibiting pure tone audiometric thresholds of $25 \mathrm{~dB}$ HL or better for both right and left ears.

With the exception of the left ear inattentiveness index (Inattentiveness right ear $U=144$, $\mathrm{p}=0.001$ ), which showed a similar distribution of results between groups, both impulsivity and inattentiveness indexes were higher for the MCI as compared to the control group (right ear $\mathrm{U}=149$, $p=0.001$, left ear $U=137.5, p=0.004)$. When statistics were run for all participants collapsed across both groups, correlations between GIN Impulsivity and Inattentiveness indexes and SinBRE were statistically significant $(r=0.496, p=0.009$ for Impulsivity right ear results; $r=0.413, p=0.036$ for Inattentiveness right ear results). When statistics were run only for participants with PTA $\leqq 25 \mathrm{~dB}$ HL, RGDT and GIN right ear thresholds correlated for both groups $(r=0.6, p=0.01)$.

\section{Discussion}

The findings of the present study demonstrate the poorer speech recognition in babble performance of patients diagnosed with MCI compared to age-matched normal controls, which is consistent with other published reports (Idrizbegovic et al, 2011; Gates et al, 2002) showing similar effects in population approach studies. Of interest, the two derived measures to estimate auditory attention and executive function (inattentiveness and impulsivity) within task performance also revealed more impaired performance in the MCI group in most measures with the exception of the left ear inattentiveness index relative to the GIN results in which similar distributions were seen between groups. These 
results highlight the complicated interaction between auditory processing and attention. Correlation of these two derived measures with SinB performance was evident when collapsing data across both groups but not when isolating MCI group results. This suggests that the observed correlation is a function of age rather than related to MCI characteristics. To our knowledge, these are novel findings that may help us understand the interplay of cognition, auditory processing and aging in individuals diagnosed with MCI.

Everyday listening is known to deteriorate with age (Sheft et al, 2015) and has been linked to a declines in a number of domains, including hearing sensitivity, which reflects peripheral hearing (Jerger et al, 1989), central auditory processing due to changes in the central auditory nervous system (Gates et al, 1995), cognition, and/or to a multifactorial central presbycusis incorporating peripheral and central auditory function, as well as general aging decline (Humes et al, 2012). In the present study, hearing sensitivity (i.e., degree of hearing loss) was comparable across the MCI and the normal control group; however, speech recognition in babble was poorer in the MCI group. These findings do not identify the source(s) of the two groups' performance, leaving unanswered whether a central auditory deficit or a general/global cognitive deficit underlies the two group's auditory performance. The MCI group's greater impulsivity of responses during GIN testing reflects a cognitive contributing factor that did not seem to correlate with the MCI group's speech in babble performance. This suggests that despite the GIN's low cognitive load compared to other temporal measurements (e.g., RGAP), cognitive load might still be sufficient to influence results near-threshold. The inattentiveness index differentiated between MCI and control groups only for right ear responses. This ear specific finding suggests an auditory processing domain effect or a connectivity issue among auditory, attention and executive areas of the brain.

The present study provides further evidence that the decline in peripheral hearing sensitivity cannot be solely responsible for the speech recognition in babble perceptual difficulties exhibited by the MCI group. In a population longitudinal study of dementia and aging, Gates, Anderson, McCurry, Feeney and Larson (2011) verified that deficits in central auditory processing (as assessed on the 
basis of a speech-based test battery of dichotic tests and monaural speech in competition tests) appear to be a precursor of Alzheimer's disease, at least in a subgroup of patients. This dementia study assessed risk factors for developing Alzheimer's disease in patients who were not diagnosed with MCI. Gates et al. (2011) argued that cortical areas neurodegeneration may be reflected on central auditory processing testing at an earlier stage than cognitive screening tests and, consequently, central auditory processing evaluation may be a valuable screening tool for dementia and assist in earlier diagnosis and treatment. A previous study by Gates, Beiser, Rees, D'Agostino and Wolf (2002) reported that $46.7 \%$ of an elderly population diagnosed with CAPD developed Alzheimer's disease 5.1 to 7.9 years later. An interesting finding of the Gates study was that the poorer the speech in noise/competition recognition abilities, the shorter the period from auditory processing evaluation to Alzheimer's diagnosis, possibly revealing a gradual deterioration of speech perception. This finding suggests the importance of early detection of a central auditory processing disorder, which, if managed may have a positive impact on the patient with dementia's communication deficits. Unilateral perceptual deficits of speech recognition in noise/competition also were evident in Gates et al. (2002). Both studies by Gates et al. $(2002 ; 2011)$ focused on the possibility of using central auditory processing evaluation as an index to detect elderly who are at a higher risk for developing cognitive decline. The question remained which central auditory processing test would be most effective for that screening. The results of the present study on two types of central auditory processing tests (speech in babble and temporal resolution) may offer some insights as to which measures might best screen for the early identification of cognitive decline.

Speech in babble perceptual ability is driven by bottom-up mechanisms of separating frequency and intensity spectrums of the target speech stimuli, as well as top-down mechanisms of selecting the speech target out of the competing babble as an auditory object in the process of auditory scene analysis (Griffiths and Warren, 2015; Spyridakou and Bamiou, 2015). Temporal resolution is defined as the shortest time period over which discrimination of two signals takes place during perception of auditory stimuli. This auditory processing ability to discriminate rapid stimulus changes is 
essential to receptive language skills and communication and has been shown to be impaired in older adults with aging rather than hearing loss indicated as the contributing factor (Humes et al, 2012; Iliadou et al, 2014). The deteriorating age effect observed in this study on the GIN was also reported by Murphy, Dillon, Schochat, Musiek, Bamiou (2016 personal communication) who examined adults similar in age (71-85) to those of the present study with no cognitive, psychological or neurological issues and with symmetrical ( $<10 \mathrm{~dB}$ ear threshold difference for each tested frequency) and presbycusis not exceeding $40 \mathrm{~dB}$ HL per each octave frequency from 250 to $8000 \mathrm{~Hz}$. Murphy, Dillon, Schochat, Musiek, Bamiou (2016 personal communication) obtained GIN thresholds of $8.19 \mathrm{~ms}$ \pm 3.5 (age group 61-70) and $11.47 \mathrm{~ms} \pm 4.86$.

The results of the present study show that both temporal resolution tests (GIN and RGDT) were efficient in differentiating the MCI group from the age matched normal control group, although the gap detection thresholds obtained using the GIN were shorter than those obtained for the RGDT, a finding seen in other clinical populations, including children with CAPD and first episode psychosis patients (Iliadou et al, 2014). This difference in gap thresholds is related to the differences in the two tests' stimuli and procedures (Chermak et al, 2005), rather than due to the characteristics of the MCI group or the elderly population in general. The MCI group's longer gap detection thresholds may contribute to their poorer speech in babble scores. While both the GIN and the RGDT differentiated the two groups, the monaural administration feature of the GIN allows one to examine possible ear differences, which could assist in differential diagnosis and determining the underlying source of MCI patients' deficits (e.g., cognitive v. auditory sources). .

In the present study, even though both ear results were different across the two groups (MCI vs Normal Controls), the right ear gap detection thresholds showed a clear cut differentiation between the two groups and no overlap of values, whereas the left ear results while still differentiating the two groups exhibited a small degree of overlap around $8 \mathrm{msec}$ threshold values. While this right ear effect must be interpreted with caution and studied more extensively with a larger group of MCI patients, the present authors speculate that it might be linked to a left hemisphere auditory processing deficit 
which would be consistent with Singh et al.'s (2006) report showing enhanced cortical thinning in the left hemisphere in MCI and Alzheimer patients. The cortical thinning was not limited to the temporal lobe involving the medial temporal lobe together with regions of the frontal and parietal cortices with decreased thickness documented when comparing healthy elderly brains with patients diagnosed with MCI. This general left hemisphere cortical thinning may explain the right, but not left ear inattentiveness derived score differences between MCI and control groups in the present study. In comparing MCI and Alzheimer's disease, the trend of a significant thinning extending into the lateral temporal lobe (especially seen in the left hemisphere) in the context of general cortical thinning has been evident (Singh et al, 2006). The present behavioral results may allow inferences about the more pronounced left (versus right) temporal cortical thinning in MCI patients, illustrating the promise of auditory temporal processing evaluation in the early diagnosis of dementia. Indeed, Gates et al. (2011) proposed screening older adults with hearing or listening in noise complaints as part of the evaluation for developing dementia; however, they did not measure temporal resolution in their study.

\section{Summary and Conclusions}

The present study demonstrates that measures of temporal resolution (in particular the GIN which provides for interaural comparisons) may be used not only to screen for CAPD in the elderly population, but to provide indirect evidence of hemispheric functionality through ear asymmetries in gap detection thresholds results. The patients with MCI participating in this study were able to successfully follow instructions and complete a brief auditory processing evaluation of speech recognition in babble and temporal resolution. Poorer auditory processing performance was evident in all tests administered to the MCI group when compared with an age matched normal control group, as 
were most of the within auditory task derived cognitive performance indexes. The GIN right ear gap detection thresholds exhibited greater deterioration for the MCI group vs the normal controls. This difference may be an indirect, early sign of the enhanced cortical thinning of the left temporal lobe demonstrated in the progression of the MCI to Alzheimer's disease. The results of the present study lends support to proposals (Gates et al, 2002; Gates et al, 2011) to include central auditory processing evaluation in the elderly population as a promising tool to potentially achieve earlier diagnosis of dementia. These results also demonstrate the potential value of GIN as an indirect screen of early, left temporal cortical thinning associated with the transition between MCI and Alzheimer's disease.

\section{References}

Akeroyd MA. (2008) Are individual differences in speech reception related to individual differences in cognitive ability? A survey of twenty experimental studies with normal and hearing-impaired adults. Int J Audiol 47(SUP2):S53-S71.

American Academy of Audiology (2010). Diagnosis, treatment and management of children and adults with central auditory processing disorder. Retrieved from www.audiology.org/resources/documentlibrary/Documents/CAPD\%20Guidelines\%208-2010.pdf 
American Speech-Language-Hearing Association (2005). Central auditory processing disorders. Retrieved from www.asha.org/members/deskref-journals/deskref/default, accessed June 2008.

Barnes LL, Mendes De Leon CF, Wilson RS, Bienias JL, Evans DA. (2004) Social resources and cognitive decline in a population of older African Americans and whites. Neurology 63(12):23222326.

Bennett DA, Schneider JA, Tang Y, Arnold SE, Wilson RS. (2006) The effect of social networks on the relation between Alzheimer's disease pathology and level of cognitive function in old people: A longitudinal cohort study. Lancet Neurology 5(5):406-412.

British Society of Audiology (2011). Practice Guide. An overview of current management of auditory processing disorder (APD). Retrieved from http://www.thebsa.org.uk/wpcontent/uploads/2014/04/BSA_APD_Management_1Aug11_FINAL_amended17Oct11.pdf, accessed August 2011.

Chermak GD, Lee J. (2005) Comparison of children's performance on four tests of temporal resolution. J Am Acad Audiol 16:554 - 563.

Chermak GD, Musiek F. (1997) Central auditory processing disorders: New perspectives. San Diego: Singular.

Cramer D, Howitt D. (2004) The SAGE dictionary of statistics. London SAGE. 
Dupuis K, Pichora-Fuller MK, Chasteen AL, Marchuk V, Singh G, Smith SL. (2015) Effects of hearing and vision impairments on the Montreal Cognitive Assessment. Neuropsychol Dev Cogn B Aging Neuropsychol Cogn 22(4):413-437.

Doane DP, Seward LE. (2002) Measuring skewness: A forgotten statistic? J Stat Educ 2011; 19(2).

Elangovan S, Stuart A. (2008) Natural boundaries in gap detection are related to categorical perception of stop consonants. Ear Hear 29:761-774.

Gates GA, Beiser A, Rees TS, D'Agostino RB, Wolf PA. Central auditory dysfunction may precede the onset of clinical dementia in people with probable Alzheimer's disease. J Am Geriatr Soc 50(3):482-488.

Folstein MF, Folstein SE, McHugh PR. (1975) "Mini-mental state". A practical method for grading the cognitive state of patients for the clinician. J Psychiatr Res 12(3):189-198.

Ferrand CT. (2006) Relationship between masking levels and phonatory stability in normal-speaking women. J Voice 20(2):223-228.

Gates GA, Cooper JC Jr, Kannel WB, Miller NJ. (1990) Hearing in the elderly: The Framingham cohort, 1983-1985 part 1. Basic audiometric test results. Ear Hear 11(4):247-256.

Gussekloo J, De Craen AJM, Oduber C, Van Boxtel MPJ, Westendorp RGJ. (2005) Sensory impairment and cognitive functioning in oldest-old subjects: The Leiden 85+ study. Am J Geriatr Psychiatry 13(9):781-786. 
Gallacher J, Ilubaera V, Ben-Shlomo Y, et al. (2012) Auditory threshold, phonologic demand, and incident dementia. Neurology 79(15):1583-1590.

Gatehouse S, Naylor G, Elberling C. (2006) Linear and nonlinear hearing aid fittings - 2. patterns of candidature. Int J Audiol 45(3), 153-171.

Gates GA, Karzon RK, Garcia P, et al. (1995) Auditory dysfunction in aging and senile dementia of the Alzheimer's type. Arch Neurol 52(6):626-634.

Gates GA, Anderson ML, McCurry SM, Feeney MP, Larson EB. (2011) Central auditory dysfunction as a harbinger of Alzheimer dementia. Arch Otolaryngol Head Neck Surg 137(4):390-395.

Griffiths TD, Warren JD. (2015) What is an auditory object? Nat Rev Neurosci 2004; 5(11):887-892.

Hoops S, Nazem S, Siderowf AD, et al. (2009) Validity of the MoCA and MMSE in the detection of MCI and dementia in Parkinson disease. Neurology 73(21):1738-1745.

Humes LE, Dubno JR, Gordon-Salant S, et al. (2012) Central presbycusis: A review and evaluation of the evidence. J Am Acad Audiol 23(8):635-666.

Idrizbegovic E, Hederstierna C, Dahlquist M, Nordstrom CK, Jelic V, Rosenhall U. (2011) Central auditory function in early Alzheimer's disease and in mild cognitive impairment. Age Ageing 40(2):249-254.

Iliadou V. (2013) Measuring Speech in noise issues in school aged children. Poster Presentation. 3rd Congress of Bio-Psycho-Social approach in Health Management, Thessaloniki. 
Iliadou V, Kaprinis S. (2003) Clinical psychoacoustic in Alzheimer's disease central auditory processing disorders and speech deterioration. Ann Gen Hosp Psychiatry 22(1):12.

Iliadou V, Fourakis M, Vakalos A, Hawks JW, Kaprinis G. (2006) Bi-syllabic, modern Greek word lists for use in word recognition tests. Int J Audiol 45(2):74-82.

Iliadou V, Bamiou DE, Kaprinis S, Kandylis D, Kaprinis G. (2009) Auditory processing disorders in children suspected of learning disabilities-A need for screening? Int J Pediatr Otorhinolaryngol 2009; 73(7):1029-1034.

Iliadou V, Bamiou DE, Chermak GD, Nimatoudis I. (2014) Comparison of two tests of auditory temporal resolution in children with central auditory processing disorder, adults with psychosis, and adult professional musicians. Int J Audiol 53(8):507-513.

Jorgensen LE, Palmer CV, Pratt S, Erickson KI, Moncrieff D. (2016) The Effect of Decreased Audibility on MMSE Performance: A Measure Commonly Used for Diagnosing Dementia. J Am Acad Audiol 27(4):311-323.

Jerger J, Jerger S, Oliver T, Pirozzolo F. (1989) Speech understanding in the elderly. Ear Hearing 10(2):79-89.

Keith R. Random Gap Detection Test, Auditec, St. Louis, MO 2000. 
Lindenberger U, Scherer H, Baltes PB. (2001) The strong connection between sensory and cognitive performance in old age: not due to sensory acuity reductions operating during cognitive assessment. Psychol Aging 6(2):196-205.

Lin FR. (2011) Hearing loss and cognition among older adults in the United States. J Gerontol A Biol Sci Med Sci 66(10):1131-1136.

Lin FR, Yaffe K, Xia J, et al. (2013) Hearing loss and cognitive decline in older adults. JAMA Intern Med 173(4):293-299.

Lin FR, Ferrucci L, An Y, et al. (2014) Association of hearing impairment with brain volume changes in older adults. Neuroimage 90:84-92.

Lister J, Besing J, Koehnke J. (2002) Effects of age and frequency disparity on gap discrimination. J Acoust Soc Am 111:2793-2800.

Lister JJ, Roberts RA, Lister FL. (2011) An adaptive clinical test of temporal resolution: Age effects. Int J Audiol 50:367-374.

McCoy SL, Tun PA, Cox LC, Colangelo M, Stewart RA, Wingfield A. (2005) Hearing loss and perceptual effort: Downstream effects on older adults' memory for speech. Q J Exp Psychol A $58(1): 22-33$.

Moore BCJ, Peters RW, Glasberg BR. (1992) Detection of temporal gaps in sinusoids by elderly subjects with and without hearing loss. J Acoust Soc Am 92(4 I):1923-1932. 
Musiek FE, Shinn JB, Jirsa R, Bamiou DE, Baran JA, Zaida E. (2005) GIN (gaps-in-noise) test performance in subjects with confirmed central auditory nervous system involvement. Ear Hearing 26(6):608-618.

Morris JC. (1993) The Clinical Dementia Rating (CDR): Current version and scoring rules. Neurology 43(11):2412-2414.

Nasreddine ZS, Phillips NA, Bedirian V, et al. (2005) The Montreal cognitive assessment, MoCA: A brief screening tool for mild cognitive impairment. J Am Geriatr Soc 53(4):695-699.

O'Bryant SE, Lacritz LH. (2010) Hall, J, et al. Validation of the new interpretive guidelines for the clinical dementia rating scale sum of boxes score in the national Alzheimer's coordinating center database. Arch Neurol 67(6):746-749.

Phillips DP. (2002) Central auditory system and central auditory processing disorders: Some conceptual issues. Semin Hear 23:251-261.

Pichora-Fuller MK, Souza PE. (2003) Effects of aging on auditory processing of speech. Int J Audiol 42(SUP2):2S11-2S16.

Popovic IM, Seric V, Demarin V. (2007) Mild cognitive impairment in symptomatic and asymptomatic cerebrovascular disease. J Neurol Sci 257(1-2):185-193.

Pendlebury ST, Cuthbertson FC, Welch SJ, Mehta Z, Rothwell PM. (2010) Underestimation of cognitive impairment by Mini-Mental State Examination versus the Montreal Cognitive Assessment in patients with transient ischemic attack and stroke: a population-based study. Stroke 41(6):1290-1293. 
Quaranta N, Coppola F, Casulli M, et al. (2015) The prevalence of peripheral and central hearing impairment and its relation to cognition in older adults. Audiol Neurotol 19:10-14.

Rahman TTA, Mohamed ST, Albanouby MH, Bekhet HF. (2011) Central auditory processing in elderly with mild cognitive impairment. Geriatr Gerontol Int 11(3):304-308.

Ronnberg J. (2003) Cognition in the hearing impaired and deaf as a bridge between signal and dialogue: A framework and a model. Int J Audiol 42(SUP1):S68-S76.

Shinn JB, Chermak GD, Musiek FE. GIN (gaps-in-noise) performance in the pediatric population. J Am Acad Audiol 20(4):229-238.

Snell KB, Frisina DR. (2000) Relationships among age-related differences in gap detection and word recognition. J Acoust Soc Am 107(3):1615-1626.

Sidiras C, Iliadou V, Chermak GD, Nimatoudis I. (2016) Assessment of Functional Hearing in GreekSpeaking Children Diagnosed with Central Auditory Processing Disorder. J Am Acad Audiol 27(5):395-405.

Sheft S, Shafiro V, Wang E, Barnes LL, Shah RC. (2015) Relationship between auditory and cognitive abilities in older adults. PLoS ONE 10(8):e0134330.

Singh V, Chertkow H, Lerch JP, Evans AC, Dorr AE, Kabani NJ. (2006) Spatial patterns of cortical thinning in mild cognitive impairment and Alzheimer's disease. Brain 129(11):2885-2893. 
Spyridakou C, Bamiou DE. Need of speech-in-noise testing to assess listening difficulties in older adults. Hearing Balance Commun 13(2):65-76.

Weinstein BE. (1986) Hearing loss and Senile Dementia in the Institutionalized Elderly. Clin Gerontol 4:3-15.

Winblad B, Palmer K, Kivipelto M, et al. (2004) Mild cognitive impairment — beyond controversies, towards a consensus: report of the International Working Group on Mild Cognitive Impairment. J Intern Med 256:240-6.

Yesavage JA, Brink TL, Rose TL, et al. (1982-83) Development and validation of a geriatric depression screening scale: a preliminary report. J Psychiatr Res 17(1):37-49. 